Norm inflation for generalized magneto-hydrodynamic system

This content has been downloaded from IOPscience. Please scroll down to see the full text. 2015 Nonlinearity 28129

(http://iopscience.iop.org/0951-7715/28/1/129)

View the table of contents for this issue, or go to the journal homepage for more

Download details:

IP Address: 131.193.153.236

This content was downloaded on 25/02/2015 at 20:58

Please note that terms and conditions apply. 


\title{
Norm inflation for generalized magneto-hydrodynamic system
}

\author{
Alexey Cheskidov and Mimi Dai \\ Department of Mathematics, Statistics and Computer Science, University of Illinois Chicago, \\ Chicago, IL 60607, USA \\ E-mail: acheskid@math.uic.edu and mdai@uic.edu
}

Received 8 February 2014, revised 30 October 2014

Accepted for publication 6 November 2014

Published 8 December 2014

Recommended by K Ohkitani

\begin{abstract}
We consider the incompressible magneto-hydrodynamic system with fractional powers of the Laplacian in the three-dimensional case. We discover a wide range of spaces where the norm inflation occurs and hence small initial data results are out of reach. The norm inflation occurs not only in scaling invariant (critical) spaces, but also in supercritical and, surprisingly, subcritical ones.
\end{abstract}

Keywords: norm inflation, generalized magneto-hydrodynamic system, Besov spaces, interactions of plane waves Mathematics Subject Classification: 76D03, 35Q35

\section{Introduction}

In this paper we study the three dimensional incompressible magneto-hydrodynamic (MHD) system with fractional powers of the Laplacian:

$$
\begin{aligned}
& u_{t}+(u \cdot \nabla) u-(b \cdot \nabla) b+\nabla p=-v(-\Delta)^{\alpha_{1}} u, \\
& b_{t}+(u \cdot \nabla) b-(b \cdot \nabla) u=-\mu(-\Delta)^{\alpha_{2}} b, \\
& u(x, 0)=u_{0}, \quad b(x, 0)=b_{0},
\end{aligned}
$$

where $x \in \mathbb{R}^{3}, t \geqslant 0, u$ is the fluid velocity, $p$ is the pressure of the fluid and $b$ is the magnetic field. The parameter $v$ denotes the kinematic viscosity coefficient of the fluid and $\mu$ denotes the reciprocal of the magnetic Reynolds number. The initial data $u_{0}$ and $b_{0}$ are divergence free. The power $\alpha_{1}=\alpha_{2}=1$ corresponds to the classical MHD system. A vast amount of literature has been devoted to these equations, for background we refer the readers to [3, 7, 11-14]. 
Solutions to the generalized MHD system (1.1) are scaling invariant when $\alpha_{1}=\alpha_{2}=$ $\alpha>0$. In this case, if $(u(x, t), p(x, t), b(x, t))$ solves system (1.1) with the initial data $\left(u_{0}(x), b_{0}(x)\right)$, then

$$
\begin{aligned}
& u_{\lambda}(x, t)=\lambda^{2 \alpha-1} u\left(\lambda x, \lambda^{2 \alpha} t\right), \quad p_{\lambda}(x, t)=\lambda^{2(2 \alpha-1)} p\left(\lambda x, \lambda^{2 \alpha} t\right) \\
& b_{\lambda}(x, t)=\lambda^{2 \alpha-1} b\left(\lambda x, \lambda^{2 \alpha} t\right)
\end{aligned}
$$

solves system (1.1) with the initial data

$$
u_{0 \lambda}=\lambda^{2 \alpha-1} u_{0}(\lambda x), b_{0 \lambda}=\lambda^{2 \alpha-1} b_{0}(\lambda x)
$$

A space that is invariant under the above scaling is called a critical space. The largest critical space for the generalized MHD system (1.1) when $\alpha_{1}=\alpha_{2}=\alpha$ is the Besov space $\dot{B}_{\infty, \infty}^{1-2 \alpha}$ (see [2]).

In the case $b=0,(1.1)$ reduces to the generalized Navier-Stokes system that has been studied extensively. Since the global regularity is only known for $\alpha_{1} \geqslant 5 / 4$, the question of global well-posedness in various critical spaces is of great interest. In the classical case $\alpha_{1}=1$ the best small initial data result is due to Koch and Tataru. In [8], they established the global well-posedness of the Navier-Stokes equations with small initial data in the space $B M O^{-1}$. In the case $\alpha_{1} \in(1 / 2,1)$ the situation is better as $\mathrm{Yu}$ and Zhai [16] showed global well-posedness for small initial data in the largest critical space $\dot{B}_{\infty, \infty}^{1-2 \alpha_{1}}$, which is out of reach for $\alpha_{1} \in[1,5 / 4)$. Obstacles here are illustrated by some Ill-posedness results: Bourgain and Pavlović [1] showed the norm inflation for the classical Navier-Stokes equations in $\dot{B}_{\infty, \infty}^{-1}$ in case $\alpha_{1}=1$, Cheskidov and Shvydkoy [5,6] proved the existence of discontinuous LerayHopf solutions of the Navier-Stokes equations in $\dot{B}_{\infty, \infty}^{1-2 \alpha_{1}}$ with arbitrarily small initial data for $\alpha_{1} \in[1,5 / 4)$, and Yoneda [15] showed the norm inflation for the classical Navier-Stokes equation in a generalized Besov space near $\mathrm{BMO}^{-1}$.

Recently, Cheskidov and Dai [4] showed the norm inflation in subcritical spaces $\dot{B}_{\infty, \infty}^{-s}$ for all $s \geqslant \alpha_{1}, \alpha_{1} \geqslant 1$. This provides a wide range of spaces where a small initial data result is not expected. Note that the natural space for the norm inflation $B_{\infty, \infty}^{-\alpha_{1}}$ is only scaling invariant in the classical case $\alpha_{1}=1$, and is subcritical for $\alpha_{1}>1$. This explains why small initial data results are only available for $\alpha_{1}<1$.

The goal of this paper is to find natural norm inflation spaces for the generalized MHD system (1.1) and show that in general they are not scaling invariant, even in the classical case. Namely, we prove that

Theorem 1.1. Let $\alpha_{1}, \alpha_{2} \geqslant 1$. Assume $\theta_{1}+\theta_{2}=2 \alpha_{2}$ for $\theta_{1}, \theta_{2}>0$. For any $\delta>0$ there exists a smooth space-periodic solution $(u(t), b(t))$ of $(1.1)$ with period $2 \pi$ and the initial data

$$
\|u(0)\|_{\dot{B}_{\infty, \infty}^{-\theta_{1}}}+\|b(0)\|_{\dot{B}_{\infty, \infty}^{-\theta_{2}}} \lesssim \delta
$$

that satisfies, for some $0<T<\delta$ and all $s>0$,

$$
\|b(T)\|_{\dot{B}_{\infty, \infty}^{-s}} \gtrsim \frac{1}{\delta}
$$

We refer the readers to the beginning of section of Preliminaries for the definition of the symbol $\lesssim$. Note that the homogeneous and non-homogeneous Besov norms are equivalent for periodic functions. Therefore, for the space-periodic solution in theorem 1.1 we have

$$
\|b(0)\|_{\dot{B}_{\infty, \infty}^{-s}} \lesssim\|b(0)\|_{B_{\infty, \infty}^{-s}} \lesssim\|b(0)\|_{\dot{B}_{\infty, \infty}^{-\theta_{2}}} \lesssim \delta \quad \text { for all } \quad s \geqslant \theta_{2}
$$

which means that the norm inflation for $b$ occurs in all the spaces $\dot{B}_{\infty, \infty}^{-s}, s>0$. More precisely, we have the following. 
Corollary 1.2. Let $\alpha_{1}, \alpha_{2} \geqslant 1$. For any $s>0, \delta>0$, and positive $\theta$ such that $\theta \geqslant 2 \alpha_{2}-s$ there exists a smooth space-periodic solution $(u(t), b(t))$ of $(1.1)$ with the initial data

$$
\|u(0)\|_{\dot{B}_{\infty, \infty}^{-\theta}}+\|b(0)\|_{\dot{B}_{\infty, \infty}^{-s}} \lesssim \delta
$$

that satisfies, for some $0<T<\delta$,

$$
\|b(T)\|_{\dot{B}_{\infty, \infty}^{-s}} \gtrsim \frac{1}{\delta}
$$

In a previous work of Dai et al [7], the authors obtained norm inflations for the classical MHD system, i.e. $\alpha_{1}=\alpha_{2}=1$ in (1.1). More precisely, the main result reads that initial data can be constructed such that

$$
\|u(0)\|_{\dot{B}_{\infty, \infty}^{-1}}+\|b(0)\|_{\dot{B}_{\infty, \infty}^{-1}} \lesssim \delta,
$$

while

$$
\|b(T)\|_{\dot{B}_{\infty, \infty}^{-1}} \gtrsim \frac{1}{\delta}, \quad \text { for some } 0<T<\delta .
$$

One can see the above result is a particular case of corollary 1.2 corresponding to $\alpha_{1}=\alpha_{2}=$ $\theta=s=1$. In fact, taking $\alpha_{1}=\alpha_{2}=1$, corollary 1.2 guarantees that the magnetic field $b$ develops norm inflation in all the spaces $\dot{B}_{\infty, \infty}^{-s}, s>0$, not only the critical one $\dot{B}_{\infty, \infty}^{1-2 \alpha_{1}}$.

We point out another difference with [7]. For the classical MHD system, the proof of the norm inflation is based on the continuity of the bilinear operator (see (2.7) in [7]) associated with the heat kernel on certain Koch-Tataru space to control the nonlinear interactions in the error terms. For the bilinear operator corresponding to the fractional heat kernel, as discussed in [4], the continuity property on a modified Koch-Tataru adapted space is not available. Instead, the bilinear operator is estimated in $L^{\infty}$ in order to control the error terms in section 4.3.

The rest of the paper is organized as follows. In section 2 we introduce some notations that shall be used throughout the paper and some auxiliary results; in section 3 we describe how the diffusions of plane waves interact in the fractional MHD system; in section 4 we devote to proving theorem 1.1 .

\section{Preliminaries}

\subsection{Notation}

We denote by $A \lesssim B$ an estimate of the form $A \leqslant C B$ with some absolute constant $C$, and by $A \sim B$ an estimate of the form $C_{1} B \leqslant A \leqslant C_{2} B$ with some absolute constants $C_{1}, C_{2}$. For simplification of the notation, we denote $\|\cdot\|_{p}=\|\cdot\|_{L^{p}}$.

\subsection{Norm of Besov spaces}

We recall the definitions of norms for the homogeneous and non-homogeneous Besov spaces $\dot{B}_{\infty, \infty}^{-s}$ and $B_{\infty, \infty}^{-s}($ see [9])

$$
\begin{aligned}
& \|f\|_{\dot{B}_{\infty, \infty}^{-s}}=\sup _{t>0} t^{\frac{s}{2 \alpha}}\left\|\mathrm{e}^{-t(-\Delta)^{\alpha}} f\right\|_{L^{\infty}}, \\
& \|f\|_{B_{\infty, \infty}^{-s}}=\sup _{0<t<1} t^{\frac{s}{2 \alpha}}\left\|\mathrm{e}^{-t(-\Delta)^{\alpha}} f\right\|_{L^{\infty}} .
\end{aligned}
$$

Note that for the periodic functions the homogeneous and non-homogeneous norms are equivalent (see [10]). It is then easy to observe that

$$
\|f\|_{\dot{B}_{\infty, \infty}^{-s}\left(\mathbb{T}^{3}\right)} \leqslant\|f\|_{L^{\infty}\left(\mathbb{T}^{3}\right)},
$$

since $\left\|\mathrm{e}^{-t(-\Delta)^{\alpha}} f\right\|_{L^{\infty}} \leqslant\|f\|_{L^{\infty}}$. 


\subsection{Bilinear operator}

Let $\mathbb{P}$ denote the projection on divergence-free vector fields, which acts on a function $\phi$ as

$$
\mathbb{P}(\phi)=\phi+\nabla \cdot(-\triangle)^{-1} \operatorname{div} \phi .
$$

Define the bilinear operator for $\alpha>1$

$$
\mathcal{B}_{\alpha}(u, v)=\int_{0}^{t} \mathrm{e}^{-(t-\tau)(-\Delta)^{\alpha}} \mathbb{P} \nabla \cdot(u \otimes v) \mathrm{d} \tau .
$$

As shown in [4] the following estimate for the bilinear operator $\mathcal{B}_{\alpha}$ holds.

Lemma 2.1. Let $u, v \in L^{1}\left(0, T ; L^{\infty}\right)$ be such that $u \otimes v \in L^{1}\left(0, T ; L^{\infty}\right)$. Then for all $\alpha>0$, the bilinear operator satisfies

$$
\left\|\mathcal{B}_{\alpha}(u, v)\right\|_{\infty} \lesssim \int_{0}^{t} \frac{1}{(t-\tau)^{1 / 2 \alpha}}\|u(\tau)\|_{\infty}\|v(\tau)\|_{\infty} \mathrm{d} \tau .
$$

\section{Interactions of plane waves}

\subsection{The first iteration approximation of a mild solution}

Let $(u, b)$ be a solution to (1.1). We write it in the form

$$
\begin{aligned}
& u=\mathrm{e}^{-t(-\Delta)^{\alpha_{1}}} u_{0}-u_{1}+y, \\
& b=\mathrm{e}^{-t(-\Delta)^{\alpha_{2}}} b_{0}-b_{1}+z,
\end{aligned}
$$

where

$$
\begin{aligned}
& u_{1}(x, t)= \mathcal{B}_{\alpha_{1}}\left(\mathrm{e}^{-t(-\Delta)^{\alpha_{1}}} u_{0}(x), \mathrm{e}^{-t(-\Delta)^{\alpha_{1}}} u_{0}(x)\right) \\
&-\mathcal{B}_{\alpha_{1}}\left(\mathrm{e}^{-t(-\Delta)^{\alpha_{1}}} b_{0}(x), \mathrm{e}^{-t(-\Delta)^{\alpha_{1}}} b_{0}(x)\right), \\
& b_{1}(x, t)=\mathcal{B}_{\alpha_{2}}\left(\mathrm{e}^{-t(-\Delta)^{\alpha_{2}}} u_{0}(x), \mathrm{e}^{-t(-\Delta)^{\alpha_{2}}} b_{0}(x)\right) \\
& \quad-\mathcal{B}_{\alpha_{2}}\left(\mathrm{e}^{-t(-\Delta)^{\alpha_{2}}} b_{0}(x), \mathrm{e}^{-t(-\Delta)^{\alpha_{2}}} u_{0}(x)\right) .
\end{aligned}
$$

A simple calculation shows that

$$
\begin{aligned}
& y(t)=-\int_{0}^{t} \mathrm{e}^{-(t-\tau)(-\Delta)^{\alpha_{1}}}\left[G_{0}(\tau)+G_{1}(\tau)+G_{2}(\tau)\right] \mathrm{d} \tau, \\
& z(t)=-\int_{0}^{t} \mathrm{e}^{-(t-\tau)(-\Delta)^{\alpha_{2}}}\left[K_{0}(\tau)+K_{1}(\tau)+K_{2}(\tau)\right] \mathrm{d} \tau,
\end{aligned}
$$

where

$$
\begin{aligned}
& G_{0}=\mathbb{P}\left[\left(\mathrm{e}^{-t(-\Delta)^{\alpha_{1}}} u_{0} \cdot \nabla\right) u_{1}+\left(u_{1} \cdot \nabla\right) \mathrm{e}^{-t(-\Delta)^{\alpha_{1}}} u_{0}+\left(u_{1} \cdot \nabla\right) u_{1}\right] \\
& -\mathbb{P}\left[\left(\mathrm{e}^{-t(-\Delta)^{\alpha_{1}}} b_{0} \cdot \nabla\right) b_{1}+\left(b_{1} \cdot \nabla\right) \mathrm{e}^{-t(-\Delta)^{\alpha_{1}}} b_{0}+\left(b_{1} \cdot \nabla\right) b_{1}\right], \\
& G_{1}=\mathbb{P}\left[\left(\mathrm{e}^{-t(-\Delta)^{\alpha_{1}}} u_{0} \cdot \nabla\right) y+\left(u_{1} \cdot \nabla\right) y+(y \cdot \nabla) \mathrm{e}^{-t(-\Delta)^{\alpha_{1}}} u_{0}+(y \cdot \nabla) u_{1}\right] \\
& -\mathbb{P}\left[\left(\mathrm{e}^{-t(-\Delta)^{\alpha_{1}}} b_{0} \cdot \nabla\right) z+\left(b_{1} \cdot \nabla\right) z+(z \cdot \nabla) \mathrm{e}^{-t(-\Delta)^{\alpha_{1}}} b_{0}+(z \cdot \nabla) b_{1}\right], \\
& G_{2}=\mathbb{P}[(y \cdot \nabla) y]-\mathbb{P}[(z \cdot \nabla) z], \\
& \text { and } \\
& K_{0}=\mathbb{P}\left[\left(\mathrm{e}^{-t(-\Delta)^{\alpha_{2}}} u_{0} \cdot \nabla\right) b_{1}+\left(u_{1} \cdot \nabla\right) \mathrm{e}^{-t(-\Delta)^{\alpha_{2}}} b_{0}+\left(u_{1} \cdot \nabla\right) b_{1}\right] \\
& -\mathbb{P}\left[\left(\mathrm{e}^{-t(-\Delta)^{\alpha_{2}}} b_{0} \cdot \nabla\right) u_{1}+\left(b_{1} \cdot \nabla\right) \mathrm{e}^{-t(-\Delta)^{\alpha_{2}}} u_{0}+\left(b_{1} \cdot \nabla\right) u_{1}\right], \\
& K_{1}=\mathbb{P}\left[\left(\mathrm{e}^{-t(-\Delta)^{\alpha_{2}}} u_{0} \cdot \nabla\right) z+\left(u_{1} \cdot \nabla\right) z+(y \cdot \nabla) \mathrm{e}^{-t(-\Delta)^{\alpha_{2}}} b_{0}+(y \cdot \nabla) b_{1}\right] \\
& -\mathbb{P}\left[\left(\mathrm{e}^{-t(-\Delta)^{\alpha_{2}}} b_{0} \cdot \nabla\right) y+\left(b_{1} \cdot \nabla\right) y+(z \cdot \nabla) \mathrm{e}^{-t(-\Delta)^{\alpha_{2}}} u_{0}+(z \cdot \nabla) u_{1}\right], \\
& K_{2}=\mathbb{P}[(y \cdot \nabla) z]-\mathbb{P}[(z \cdot \nabla) y] .
\end{aligned}
$$

Note that $G_{0}, K_{0}$ do not depend on $y$ or $z, G_{1}, K_{1}$ are linear, and $G_{2}, K_{2}$ are quadratic in $y$ and $z$. 
Remark 3.1. Note that although the second equation in system (1.1) has no pressure, since $u$ and $b$ are both divergence free, the term $u \cdot \nabla b-b \cdot \nabla u$ is automatically divergence free. Hence the projector $\mathbb{P}$ acting on this term does not change the second equation and hence we can write $b_{1}$ and $K_{i}$ 's as described above.

In this section we show how the diffusions of plane waves interact in the generalized MHD system. These interactions are the basis for the constructions of initial data to produce the norm inflation.

\subsection{Diffusion of a plane wave}

Suppose $k \in \mathbb{R}^{3}, v \in \mathbb{S}^{2}$ and $k \cdot v=0$. Let

$$
u_{0}=v \cos (k \cdot x) \text {. }
$$

Then $\nabla \cdot u_{0}=0$ and

$$
\mathrm{e}^{-t(-\Delta)^{\alpha}} v \cos (k \cdot x)=\mathrm{e}^{-|k|^{2 \alpha} t} v \cos (k \cdot x) .
$$

It is also important to notice that for $s>0$

$$
\|v \cos (k \cdot x)\|_{\dot{B}_{\infty, \infty}^{-s}} \sim|k|^{-s} .
$$

\subsection{Interaction of plane waves}

Now we consider the interaction of two different single plane waves. Suppose $k_{i} \in \mathbb{R}^{3}, v_{i} \in \mathbb{S}^{2}$ and $k_{i} \cdot v_{i}=0$, for $i=1,2$. Let

$$
u_{1}=\cos \left(k_{1} \cdot x\right) v_{1}, \quad u_{2}=\cos \left(k_{2} \cdot x\right) v_{2} .
$$

To simplify our calculations we assume that $k_{2} \cdot v_{1}=\frac{1}{2}$. It then follows from a straightforward calculation that

$$
\begin{aligned}
\mathcal{B}_{\alpha}\left(\mathrm{e}^{-t(-\Delta)^{\alpha}} u_{1}, \mathrm{e}^{-t(-\Delta)^{\alpha}} u_{2}\right) & \\
= & \frac{1}{4} v_{1} \sin \left(\left(k_{2}-k_{1}\right) \cdot x\right) \int_{0}^{t} \mathrm{e}^{-\left(\left|k_{1}\right|^{2 \alpha}+\left|k_{2}\right|^{2 \alpha}\right) \tau} \mathrm{e}^{-\left|k_{2}-k_{1}\right|^{2 \alpha}(t-\tau)} \mathrm{d} \tau \\
& +\frac{1}{4} v_{1} \sin \left(\left(k_{1}+k_{2}\right) \cdot x\right) \int_{0}^{t} \mathrm{e}^{-\left(\left|k_{1}\right|^{2 \alpha}+\left|k_{2}\right|^{2 \alpha}\right) \tau} \mathrm{e}^{-\left|k_{1}+k_{2}\right|^{2 \alpha}(t-\tau)} \mathrm{d} \tau .
\end{aligned}
$$

Therefore, the interaction of the two plane waves is small in $\dot{B}_{\infty, \infty}^{-s}$ if neither the sum nor the difference of their wave vectors is small in magnitude. In the contrary, the interaction is sizable in $\dot{B}_{\infty, \infty}^{-s}$ if either the sum or the difference of their wave vectors is small in magnitude.

\section{Proof of theorem 1.1}

In this section we follow the ideas from $[1,4]$ to construct initial data that produce norm inflation for solutions to the fractional MHD system. From the discussions in section 3.3 we see that the interaction of two plane waves is not enough to show the norm inflation, which requires a large number of waves. We also make sure that the initial data is space-periodic and smooth, which ensures the local existence of a smooth periodic solution to the fractional MHD system. As we control its $L^{\infty}$ norm, the solution will remain smooth until the time of the norm inflation. 


\subsection{Construction of initial data for the fractional MHD system}

For a fixed small number $\delta>0$ that we will specify later, the initial data will be as follows:

$$
\begin{aligned}
& u_{0}=r^{-\beta_{1}} \sum_{i=1}^{r}\left|k_{i}\right|^{\theta_{1}} v \cos \left(k_{i} \cdot x\right), \\
& b_{0}=r^{-\beta_{2}} \sum_{i=1}^{r}\left|k_{i}^{\prime}\right|^{\theta_{2}} v^{\prime} \cos \left(k_{i}^{\prime} \cdot x\right),
\end{aligned}
$$

where $\beta_{1}, \beta_{2}, \theta_{1}, \theta_{2}>0$ to be determined later. We expect for each $i$ the interaction of the two plane waves $v \cos \left(k_{i} \cdot x\right)$ and $v^{\prime} \cos \left(k_{i}^{\prime} \cdot x\right)$ to be sizable in $\dot{B}_{\infty, \infty}^{-s}$, while the interactions of plane waves corresponding to different indexes $i$ to be small. Hence, we choose

- Wave vectors. Let $\zeta=(1,0,0)$ and $\eta=(0,0,1)$. The wave vectors $k_{i} \in \mathbb{Z}^{3}$ are parallel to $\zeta$. Let $K$ be a large integer dependent on $r$. The magnitude of $k_{i}$ is defined by

$$
\left|k_{i}\right|=2^{i-1} K, \quad i=1,2,3, \ldots, r .
$$

The wave vectors $k_{i}^{\prime} \in \mathbb{Z}^{3}$ are defined by

$$
k_{i}^{\prime}=k_{i}+\eta \text {. }
$$

- Amplitude vectors. Let

$$
v=(0,0,1), \quad v^{\prime}=(0,1,0) .
$$

Hence

$$
k_{i} \cdot v=k_{i}^{\prime} \cdot v^{\prime}=0
$$

which ensures that the initial data is divergence free.

The initial data construction here is not the same as the one used for the classical MHD system in [7]. We still use the fact that the interaction of the high-high frequencies waves produces low frequency wave, which is the essential part to develop the norm inflation in Besov spaces with negative indexes. However, thanks to the coupling of the velocity field and the magnetic field, we have the flexibility of having more admissible values of $\beta_{1}, \beta_{2}, \theta_{1}$ and $\theta_{2}$, which allows us to obtain a wider range of spaces where the system (1.1) develops norm inflation (see theorem 1.1 and corollary 1.2).

We recall the following simple facts whose proof can be found in [4].

Lemma 4.1. Let $\gamma, \theta>0$. With the choices (4.15)-(4.17), the following holds:

$$
\begin{aligned}
& k_{i} \cdot v^{\prime}=0, \quad k_{i}^{\prime} \cdot v=1, \quad \forall \quad i=1,2, \ldots, r, \\
& \sum_{j<i}\left|k_{j}\right|^{\theta} \sim\left|k_{i-1}\right|^{\theta} \quad \text { and } \quad \sum_{j<i}\left|k_{j}^{\prime}\right|^{\theta} \sim\left|k_{i-1}^{\prime}\right|^{\theta}, \\
& \sum_{i=1}^{r}\left|k_{i}\right|^{\gamma} \mathrm{e}^{-\left|k_{i}\right|^{2 \alpha} t} \lesssim t^{-\frac{\gamma}{2 \alpha}} \quad \text { and } \sum_{i=1}^{r}\left|k_{i}^{\prime}\right|^{\gamma} \mathrm{e}^{-\left|k^{\prime}\right|^{2 \alpha} t} \lesssim t^{-\frac{\gamma}{2 \alpha}} .
\end{aligned}
$$

Next we estimate the norms of the initial data.

Lemma 4.2. Let $\left(u_{0}, b_{0}\right)$ be given in (4.14) and $\theta_{1}, \theta_{2}>0$. Then

$$
\left\|u_{0}\right\|_{\dot{B}_{\infty, \infty}^{-\theta_{1}}} \lesssim r^{-\beta_{1}}, \quad\left\|b_{0}\right\|_{\dot{B}_{\infty, \infty}^{-\theta_{2}}} \lesssim r^{-\beta_{2}}
$$


Proof. Due to (3.13), we have that,

$$
\mathrm{e}^{-t(-\Delta)^{\theta_{1}}} u_{0}=r^{-\beta_{1}} \sum_{i=1}^{r}\left|k_{i}\right|^{\theta_{1}} v \cos \left(k_{i} \cdot x\right) \mathrm{e}^{-\left|k_{i}\right|^{2 \theta_{1} t}} .
$$

Hence by lemma 4.1,

$$
\left\|u_{0}\right\|_{\dot{B}_{\infty, \infty}^{-\theta_{1}}} \sim r^{-\beta_{1}} \sup _{0<t<1} t^{\frac{1}{2}} \sum_{i=1}^{r}\left|k_{i}\right|^{\theta_{1}} \mathrm{e}^{-\left|k_{i}\right|^{2 \theta_{1}} t} \lesssim r^{-\beta_{1}} .
$$

The estimate of $b_{0}$ in $\dot{B}_{\infty, \infty}^{-\theta_{2}}$ can be obtained similarly.

Lemma 4.3. Let $\left(u_{0}, b_{0}\right)$ be given in (4.14) and $\alpha>0$. Then

$$
\left\|\mathrm{e}^{-t(-\Delta)^{\alpha}} u_{0}\right\|_{\infty} \lesssim r^{-\beta_{1}} t^{-\frac{\theta_{1}}{2 \alpha}}, \quad\left\|\mathrm{e}^{-t(-\Delta)^{\alpha}} b_{0}\right\|_{\infty} \lesssim r^{-\beta_{2}} t^{-\frac{\theta_{2}}{2 \alpha}} .
$$

Proof. By (4.22) and lemma 4.1, we infer that

$$
\left\|\mathrm{e}^{-t(-\Delta)^{\alpha}} u_{0}\right\|_{\infty} \lesssim r^{-\beta_{1}} \sum_{i=1}^{r}\left|k_{i}\right|^{\theta_{1}} \mathrm{e}^{-\left|k_{i}\right|^{2 \alpha} t} \lesssim r^{-\beta_{1}} t^{-\frac{\theta_{1}}{2 \alpha}} .
$$

The estimate for $b_{0}$ can be obtained similarly.

4.2. Analysis of $u_{1}$ and $b_{1}$

As demonstrated in section 3.1 we consider the decomposition

$$
\begin{aligned}
& u=\mathrm{e}^{-t(-\Delta)^{\alpha_{1}}} u_{0}-u_{1}+y, \\
& b=\mathrm{e}^{-t(-\Delta)^{\alpha_{2}}} b_{0}-b_{1}+z .
\end{aligned}
$$

Recall the definition (3.7) and (3.8)

$$
\begin{aligned}
u_{1}(x, t)= & \mathcal{B}_{\alpha_{1}}\left(\mathrm{e}^{-t(-\Delta)^{\alpha_{1}}} u_{0}(x), \mathrm{e}^{-t(-\Delta)^{\alpha_{1}}} u_{0}(x)\right) \\
& -\mathcal{B}_{\alpha_{1}}\left(\mathrm{e}^{-t(-\Delta)^{\alpha_{1}}} b_{0}(x), \mathrm{e}^{-t(-\Delta)^{\alpha_{1}}} b_{0}(x)\right), \\
b_{1}(x, t)= & \mathcal{B}_{\alpha_{2}}\left(\mathrm{e}^{-t(-\Delta)^{\alpha_{2}}} u_{0}(x), \mathrm{e}^{-t(-\Delta)^{\alpha_{2}}} b_{0}(x)\right) \\
& -\mathcal{B}_{\alpha_{2}}\left(\mathrm{e}^{-t(-\Delta)^{\alpha_{2}}} b_{0}(x), \mathrm{e}^{-t(-\Delta)^{\alpha_{2}}} u_{0}(x)\right) .
\end{aligned}
$$

By the fact that $k_{i} \cdot v=k_{i}^{\prime} \cdot v^{\prime}=0$ for all $i=1,2, \ldots, r$, it is immediately seen

$$
\left(\mathrm{e}^{-t(-\Delta)^{\alpha_{1}}} u_{0} \cdot \nabla\right) \mathrm{e}^{-t(-\Delta)^{\alpha_{1}}} u_{0}=\left(\mathrm{e}^{-t(-\Delta)^{\alpha_{1}}} b_{0} \cdot \nabla\right) \mathrm{e}^{-t(-\Delta)^{\alpha_{1}}} b_{0}=0,
$$

hence $u_{1} \equiv 0$. Again since $k_{i} \cdot v^{\prime}=0$ for all $i=1,2, \ldots, r$ by (4.18), it follows

$$
\left(\mathrm{e}^{-t(-\Delta)^{\alpha_{2}}} b_{0} \cdot \nabla\right) \mathrm{e}^{-t(-\Delta)^{\alpha_{2}}} u_{0}=0,
$$

hence

$$
b_{1}(x, t)=\mathcal{B}_{\alpha_{2}}\left(\mathrm{e}^{-t(-\Delta)^{\alpha_{2}}} u_{0}(x), \mathrm{e}^{-t(-\Delta)^{\alpha_{2}}} b_{0}(x)\right) .
$$


By (4.17), (4.18), (4.22) and a straightforward calculation, it follows that

$$
\begin{aligned}
\left(\mathrm{e}^{-t(-\Delta)^{\alpha_{2}}} u_{0} \cdot \nabla\right) & \mathrm{e}^{-t(-\Delta)^{\alpha_{2}}} b_{0} \\
= & -r^{-\beta_{1}-\beta_{2}} \sum_{i=1}^{r} \sum_{j=1}^{r}\left|k_{i}\right|^{\theta_{1}}\left|k_{j}^{\prime}\right|^{\theta_{2}} \mathrm{e}^{-\left(\left|k_{i}\right|^{2 \alpha_{2}}+\left|k_{j}^{\prime}\right|^{2 \alpha_{2}}\right) t} v^{\prime} \cos \left(k_{i} \cdot x\right) \sin \left(k_{j}^{\prime} \cdot x\right) \\
= & -\frac{r^{-\beta_{1}-\beta_{2}}}{2} \sum_{i=1}^{r}\left|k_{i}\right|^{\theta_{1}}\left|k_{i}^{\prime}\right|^{\theta_{2}} \mathrm{e}^{-\left(\left|k_{i}\right|^{2 \alpha_{2}}+\left|k_{i}^{\prime}\right|^{2 \alpha_{2}}\right) t} \sin (\eta \cdot x) v^{\prime} \\
& -\frac{r^{-\beta_{1}-\beta_{2}}}{2} \sum_{i \neq j}^{r}\left|k_{i}\right|^{\theta_{1}}\left|k_{j}^{\prime}\right|^{\theta_{2}} \mathrm{e}^{-\left(\left|k_{i}\right|^{2 \alpha_{2}}+\left|k_{j}^{\prime}\right|^{2 \alpha_{2}}\right) t} \sin \left(\left(k_{j}^{\prime}-k_{i}\right) \cdot x\right) v^{\prime} \\
& -\frac{r^{-\beta_{1}-\beta_{2}}}{2} \sum_{i=1}^{r} \sum_{j=1}^{r}\left|k_{i}\right|^{\theta_{1}}\left|k_{j}^{\prime}\right|^{\theta_{2}} \mathrm{e}^{-\left(\left|k_{i}\right|^{2 \alpha_{2}}+\left|k_{j}^{\prime}\right|^{2 \alpha_{2}}\right) t} \sin \left(\left(k_{j}^{\prime}+k_{i}\right) \cdot x\right) v^{\prime} \\
:= & E_{0}+E_{1}+E_{2},
\end{aligned}
$$

where we used the formula $\cos x \sin y=[\sin (x+y)-\sin (x-y)] / 2$.

Recall that $\eta \cdot v^{\prime}=0,\left(k_{j}^{\prime}+k_{i}\right) \cdot v^{\prime}=0$ and $\left(k_{j}^{\prime}-k_{i}\right) \cdot v^{\prime}=0$ for all $i, j$ due to (4.18). Hence $E_{0}, E_{1}$ and $E_{2}$ are divergence free vectors. Thus we can write

$$
\begin{aligned}
& b_{1}=\int_{0}^{t} \mathrm{e}^{-(t-\tau)(-\Delta)^{\alpha_{2}}} E_{0}(\tau) \mathrm{d} \tau+\int_{0}^{t} \mathrm{e}^{-(t-\tau)(-\Delta)^{\alpha_{2}}} E_{1}(\tau) \mathrm{d} \tau \\
& +\int_{0}^{t} \mathrm{e}^{-(t-\tau)(-\Delta)^{\alpha_{2}}} E_{2}(\tau) \mathrm{d} \tau:=b_{10}+b_{11}+b_{12} .
\end{aligned}
$$

We have the following estimates.

Lemma 4.4. Let $b_{10}$ be defined in (4.25) and $s>0$. Assume $\theta_{1}+\theta_{2}=2 \alpha_{2}$. Then

$$
\begin{aligned}
& \left\|b_{10}(\cdot, t)\right\|_{\dot{B}_{\infty, \infty}^{-s}} \gtrsim r^{1-\beta_{1}-\beta_{2}}, \quad \text { for } \quad\left|k_{1}\right|^{-2 \alpha_{2}} \leqslant t \leqslant T, \\
& \left\|b_{10}(\cdot, t)\right\|_{\infty} \lesssim r^{1-\beta_{1}-\beta_{2}}, \quad \text { for all } t>0 .
\end{aligned}
$$

Proof. From (4.24) and (4.25) it follows by a straightforward calculation

$$
\begin{aligned}
b_{10} & =-\frac{r^{-\beta_{1}-\beta_{2}}}{2} \int_{0}^{t} \sum_{i=1}^{r}\left|k_{i}\right|^{\theta_{1}}\left|k_{i}^{\prime}\right|^{\theta_{2}} \mathrm{e}^{-\left(\left|k_{i}^{\prime}\right|^{2 \alpha_{2}}+\left|k_{i}\right|^{2 \alpha_{2}}\right) \tau} \mathrm{e}^{-|\eta|^{2 \alpha_{2}(t-\tau)}} \sin (\eta \cdot x) v^{\prime} \mathrm{d} \tau \\
& =-\frac{r^{-\beta_{1}-\beta_{2}}}{2} \sin (\eta \cdot x) v^{\prime} \sum_{i=1}^{r}\left|k_{i}\right|^{\theta_{1}}\left|k_{i}^{\prime}\right|^{\theta_{2}} \mathrm{e}^{-t} \frac{1-\mathrm{e}^{-\left(\left|k_{i}^{\prime}\right|^{2 \alpha_{2}}+\left|k_{i}\right|^{2 \alpha_{2}}-1\right) t}}{\left|k_{i}^{\prime}\right|^{2 \alpha_{2}}+\left|k_{i}\right|^{2 \alpha_{2}}-1} \\
& \sim-\frac{r^{-\beta_{1}-\beta_{2}}}{2} \sin (\eta \cdot x) v^{\prime} \sum_{i=1}^{r} \mathrm{e}^{-t}\left(1-\mathrm{e}^{-\left|k_{i}\right|^{2 \alpha_{2}} t}\right),
\end{aligned}
$$

where the assumption $\theta_{1}+\theta_{2}=2 \alpha_{2}$ implies

$$
\left|k_{i}\right|^{\theta_{1}}\left|k_{i}^{\prime}\right|^{\theta_{2}} \sim\left|k_{i}^{\prime}\right|^{2 \alpha_{2}}+\left|k_{i}\right|^{2 \alpha_{2}}-1 \text {. }
$$

Hence for $\left|k_{1}\right|^{-2 \alpha_{2}} \leqslant t \leqslant T$ and $s>0$,

$$
\left\|b_{10}(\cdot, t)\right\|_{\dot{B}_{\infty, \infty}^{-s}} \gtrsim r^{-\beta_{1}-\beta_{2}} \cdot r \sup _{0<t<1} t^{\frac{s}{2 \alpha}} \mathrm{e}^{-|\eta|^{2 \alpha} t} \gtrsim r^{1-\beta_{1}-\beta_{2}} .
$$

On the other hand,

$$
\left\|b_{10}(\cdot, t)\right\|_{\infty} \lesssim \frac{r^{-\beta_{1}-\beta_{2}}}{2} \cdot r \lesssim r^{1-\beta_{1}-\beta_{2}},
$$

for all $t>0$. 
Lemma 4.5. Let $b_{11}$ and $b_{12}$ be defined in (4.25). Then

$$
\left\|b_{11}(\cdot, t)\right\|_{\infty}+\left\|b_{12}(\cdot, t)\right\|_{\infty} \lesssim r^{-\beta_{1}-\beta_{2}} t^{1-\left(\theta_{1}+\theta_{2}\right) / 2 \alpha_{2}},
$$

for all $t>0$.

Proof. Thanks to (4.24) and (4.25), it follows that

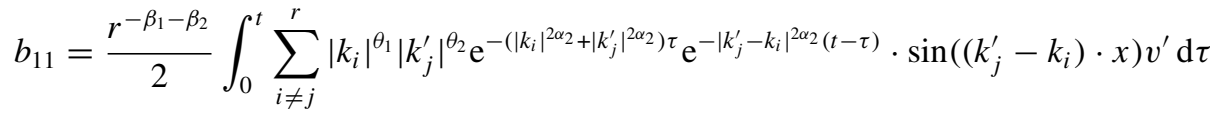

$$
\begin{aligned}
& \sim \frac{r^{-\beta_{1}-\beta_{2}}}{2} \sum_{i=1}^{r} \sum_{j<i}\left|k_{i}\right|^{\theta_{1}}\left|k_{j}^{\prime}\right|^{\theta_{2}} \mathrm{e}^{-\left|k_{i}-k_{j}^{\prime}\right|^{2 \alpha_{2}}} \frac{1-\mathrm{e}^{-\left(\left|k_{i}\right|^{2 \alpha_{2}}+\left|k_{j}^{\prime}\right|^{2 \alpha_{2}}-\left|k_{i}-k_{j}^{\prime}\right|^{2 \alpha_{2}}\right) t}}{\left|k_{i}\right|^{2 \alpha_{2}}+\left|k_{j}^{\prime}\right|^{2 \alpha_{2}}-\left|k_{i}-k_{j}^{\prime}\right|^{2 \alpha_{2}}} \\
& \cdot \sin \left(\left(k_{j}^{\prime}-k_{i}\right) \cdot x\right) v^{\prime} \\
& \sim \frac{r^{-\beta_{1}-\beta_{2}}}{2} \sum_{i=1}^{r} \sum_{j<i}\left|k_{i}\right|^{\theta_{1}}\left|k_{j}^{\prime}\right|^{\theta_{2}} t e^{-\left|k_{i}\right|^{2 \alpha_{2} t}} \sin \left(\left(k_{j}^{\prime}-k_{i}\right) \cdot x\right) v^{\prime},
\end{aligned}
$$

where we used the fact that $\frac{1-\mathrm{e}^{-x}}{x}$ is bounded for $x>0$. Hence, by (4.19) and (4.20) we infer that

$$
\begin{aligned}
\left\|b_{11}(\cdot, t)\right\|_{\infty} & \lesssim r^{-\beta_{1}-\beta_{2}} \sum_{i=1}^{r} \sum_{j<i}\left|k_{i}\right|^{\theta_{1}}\left|k_{j}^{\prime}\right|^{\theta_{2}} t \mathrm{e}^{-\left|k_{i}\right|^{2 \alpha_{2}} t} \\
& \lesssim r^{-\beta_{1}-\beta_{2}} \sum_{i=1}^{r}\left|k_{i}\right|^{\theta_{1}}\left|k_{i}^{\prime}\right|^{\theta_{2}} t \mathrm{e}^{-\left|k_{i}\right|^{2 \alpha_{2} t}} \\
& \lesssim r^{-\beta_{1}-\beta_{2}} \sum_{i=1}^{r}\left|k_{i}\right|^{\theta_{1}+\theta_{2}} t \mathrm{e}^{-\left|k_{i}\right|^{2 \alpha_{2} t}} \\
& \lesssim r^{-\beta_{1}-\beta_{2}} t^{1-\left(\theta_{1}+\theta_{2}\right) / 2 \alpha_{2}} .
\end{aligned}
$$

Similarly, we have

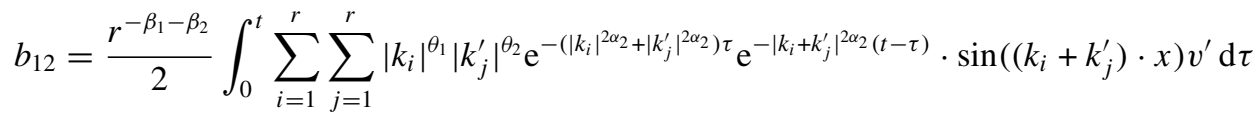

$$
\begin{aligned}
& =\frac{r^{-\beta_{1}-\beta_{2}}}{2} \sum_{i=1}^{r} \sum_{j=1}^{r}\left|k_{i}\right|^{\theta_{1}}\left|k_{j}^{\prime}\right|^{\theta_{2}} \mathrm{e}^{-\left(\left|k_{i}\right|^{2 \alpha_{2}}+\left|k_{j}^{\prime}\right|^{2 \alpha_{2}}\right) t} \frac{1-\mathrm{e}^{-\left(\left|k_{i}+k_{j}^{\prime}\right|^{2 \alpha_{2}}-\left|k_{i}\right|^{2 \alpha_{2}}-\left|k_{j}^{\prime}\right|^{2 \alpha_{2}}\right) t}}{\left|k_{i}+k_{j}^{\prime}\right|^{2 \alpha_{2}}-\left|k_{i}\right|^{2 \alpha_{2}}-\left|k_{j}^{\prime}\right|^{2 \alpha_{2}}} \\
& \cdot \sin \left(\left(k_{i}+k_{j}^{\prime}\right) \cdot x\right) v^{\prime} \\
& \sim r^{-\beta_{1}-\beta_{2}} \sum_{i=1}^{r} \sum_{j \leqslant i}\left|k_{i}\right|^{\theta_{1}}\left|k_{j}^{\prime}\right|^{\theta_{2}} t \mathrm{e}^{-\left(\left|k_{i}\right|^{2 \alpha_{2}}+\left|k_{j}^{\prime}\right|^{2 \alpha_{2}}\right) t} \sin \left(\left(k_{i}+k_{j}^{\prime}\right) \cdot x\right) v^{\prime} .
\end{aligned}
$$

Thus,

$$
\begin{aligned}
\left\|b_{12}(\cdot, t)\right\|_{\infty} & \lesssim r^{-\beta_{1}-\beta_{2}} \sum_{i=1}^{r} \sum_{j \leqslant i}\left|k_{i}\right|^{\theta_{1}}\left|k_{j}^{\prime}\right|^{\theta_{2}} t \mathrm{e}^{-\left|k_{i}\right|^{2 \alpha} t} \\
& \lesssim r^{-\beta_{1}-\beta_{2}} \sum_{i=1}^{r}\left|k_{i}\right|^{\theta_{1}+\theta_{2}} t \mathrm{e}^{-\left|k_{i}\right|^{2 \alpha} t} \\
& \lesssim r^{-\beta_{1}-\beta_{2}} t^{1-\left(\theta_{1}+\theta_{2}\right) / 2 \alpha_{2}}
\end{aligned}
$$




\subsection{Analysis of $y$ and $z$}

In this section we analyse the parts $y$ and $z$ of the solution. The idea is to control $y, z$ using the estimate (2.4) of the bilinear operator $\mathcal{B}_{\alpha}$ in the space $L^{\infty}$.

Recall from section 3.1 that

$$
\begin{aligned}
& y(t)=-\int_{0}^{t} \mathrm{e}^{-(t-\tau)(-\Delta)^{\alpha_{1}}}\left[G_{0}(\tau)+G_{1}(\tau)+G_{2}(\tau)\right] \mathrm{d} \tau, \quad t \in[0, T] . \\
& z(t)=-\int_{0}^{t} \mathrm{e}^{-(t-\tau)(-\Delta)^{\alpha_{2}}}\left[K_{0}(\tau)+K_{1}(\tau)+K_{2}(\tau)\right] \mathrm{d} \tau, \quad t \in[0, T] .
\end{aligned}
$$

Lemma 4.6. Let $\alpha_{1}, \alpha_{2} \in[1, \infty)$ and $\beta_{1}, \beta_{2} \in\left(\frac{1}{3}, \frac{1}{2}\right)$. Assume additionally that

$$
\left\{\begin{array}{l}
\theta_{1}+\theta_{2}=2 \alpha_{2}, \\
1 \leqslant \theta_{1} \leqslant 2 \alpha_{1}-1, \\
1 \leqslant \theta_{2} \leqslant 2 \alpha_{1}-1 .
\end{array}\right.
$$

Then

$$
\begin{gathered}
\|y(t)\|_{\infty}+\|z(t)\|_{\infty} \lesssim \\
r^{1-\beta_{1}-2 \beta_{2}} t^{1-\frac{1}{2 \alpha_{1}}-\frac{\theta_{2}}{2 \alpha_{1}}}+r^{2\left(1-\beta_{1}-\beta_{2}\right)} t^{1-\frac{1}{2 \alpha_{1}}} \\
+r^{1-2 \beta_{1}-\beta_{2}} t^{1-\frac{1}{2 \alpha_{2}}-\frac{\theta_{1}}{2 \alpha_{2}}}
\end{gathered}
$$

for all $0 \leqslant t \leqslant T$, provided $T$ is small and $r$ is large enough.

Proof. Recall that $u_{1}=0$. It follows from (3.11) and (4.26) that

$$
\begin{aligned}
\|y(t)\|_{\infty} \lesssim & \left\|\mathcal{B}_{\alpha_{1}}\left(\mathrm{e}^{-t(-\Delta)^{\alpha_{1}}} b_{0}, b_{1}(t)\right)\right\|_{\infty}+\left\|\mathcal{B}_{\alpha_{1}}\left(b_{1}(t), b_{1}(t)\right)\right\|_{\infty} \\
& +\left\|\mathcal{B}_{\alpha_{1}}\left(\mathrm{e}^{-t(-\Delta)^{\alpha_{1}}} u_{0}, y(t)\right)\right\|_{\infty}+\left\|\mathcal{B}_{\alpha_{1}}\left(\mathrm{e}^{-t(-\Delta)^{\alpha_{1}}} b_{0}, z(t)\right)\right\|_{\infty} \\
& +\left\|\mathcal{B}_{\alpha_{1}}\left(b_{1}(t), z(t)\right)\right\|_{\infty}+\left\|\mathcal{B}_{\alpha_{1}}(y(t), y(t))\right\|_{\infty}+\left\|\mathcal{B}_{\alpha_{1}}(z(t), z(t))\right\|_{\infty} .
\end{aligned}
$$

Applying the bilinear estimate (2.4), lemmas 4.3, 4.4, and 4.5 we infer

$$
\begin{aligned}
\left\|\mathcal{B}_{\alpha_{1}}\left(\mathrm{e}^{-t(-\Delta)^{\alpha_{1}}} b_{0}, b_{1}(t)\right)\right\|_{\infty} & \lesssim \int_{0}^{t} \frac{1}{(t-\tau)^{1 / 2 \alpha_{1}}}\left\|\mathrm{e}^{-\tau(-\Delta)^{\alpha_{1}}} b_{0}\right\|_{\infty}\left\|b_{1}(\tau)\right\|_{\infty} \mathrm{d} \tau \\
& \lesssim r^{1-\beta_{1}-2 \beta_{2}} \int_{0}^{t}(t-\tau)^{-\frac{1}{2 \alpha_{1}}} \tau^{-\frac{\theta_{2}}{2 \alpha_{1}}} \mathrm{~d} \tau \\
& \lesssim r^{1-\beta_{1}-2 \beta_{2}} t^{1-\frac{1}{2 \alpha_{1}}-\frac{\theta_{2}}{2 \alpha_{1}}},
\end{aligned}
$$

where we used the boundedness of Beta function for $\alpha>1 / 2$ and $0<\frac{\theta_{2}}{2 \alpha_{1}}<1$ :

$\int_{0}^{t}(t-\tau)^{-\frac{1}{2 \alpha_{1}}} \tau^{-\frac{\theta_{2}}{2 \alpha_{1}}} \mathrm{~d} \tau=t^{1-\frac{1}{2 \alpha_{1}}-\frac{\theta_{2}}{2 \alpha_{1}}} B\left(1-\frac{\theta_{2}}{2 \alpha_{1}}, 1-\frac{1}{2 \alpha_{1}}\right) \leqslant C t^{1-\frac{1}{2 \alpha_{1}}-\frac{\theta_{2}}{2 \alpha_{1}}}$.

Similarly, using the estimates obtained in previous two subsections, we obtain

$$
\begin{aligned}
&\left\|\mathcal{B}_{\alpha_{1}}\left(b_{1}(t), b_{1}(t)\right)\right\|_{\infty} \lesssim \int_{0}^{t} \frac{1}{(t-\tau)^{1 / 2 \alpha_{1}}}\left\|b_{1}(\tau)\right\|_{\infty}^{2} \mathrm{~d} \tau \lesssim r^{2\left(1-\beta_{1}-\beta_{2}\right)} t^{1-\frac{1}{2 \alpha_{1}}} \\
&\left\|\mathcal{B}_{\alpha_{1}}\left(\mathrm{e}^{-t(-\Delta)^{\alpha_{1}}} u_{0}, y(t)\right)\right\|_{\infty} \lesssim \int_{0}^{t} \frac{1}{(t-\tau)^{1 / 2 \alpha_{1}}}\left\|\mathrm{e}^{-\tau(-\Delta)^{\alpha_{1}}} u_{0}\right\|_{\infty}\|y(\tau)\|_{\infty} \mathrm{d} \tau \\
& \lesssim r^{-\beta_{1}} \int_{0}^{t}(t-\tau)^{-\frac{1}{2 \alpha_{1}}} \tau^{-\frac{\theta_{1}}{2 \alpha_{1}}} \mathrm{~d} \tau \sup _{0<\tau<t}\|y(\tau)\|_{\infty} \\
& \lesssim r^{-\beta_{1}} t^{1-\frac{1}{2 \alpha_{1}}-\frac{\theta_{1}}{2 \alpha_{1}}} \sup _{0<\tau<t}\|y(\tau)\|_{\infty},
\end{aligned}
$$




$$
\begin{aligned}
\left\|\mathcal{B}_{\alpha_{1}}\left(\mathrm{e}^{-t(-\Delta)^{\alpha_{1}}} b_{0}, z(t)\right)\right\|_{\infty} & \lesssim \int_{0}^{t} \frac{1}{(t-\tau)^{1 / 2 \alpha_{1}}}\left\|\mathrm{e}^{-\tau(-\Delta)^{\alpha_{1}}} b_{0}\right\|_{\infty}\|z(\tau)\|_{\infty} \mathrm{d} \tau \\
& \lesssim r^{-\beta_{2}} \int_{0}^{t}(t-\tau)^{-\frac{1}{2 \alpha_{1}}} \tau^{-\frac{\theta_{2}}{2 \alpha_{1}}} \mathrm{~d} \tau \sup _{0<\tau<t}\|z(\tau)\|_{\infty} \\
& \lesssim r^{-\beta_{2}} t^{1-\frac{1}{2 \alpha_{1}}-\frac{\theta_{2}}{2 \alpha_{1}}} \sup _{0<\tau<t}\|z(\tau)\|_{\infty},
\end{aligned}
$$

$\left\|\mathcal{B}_{\alpha_{1}}\left(b_{1}(t), z(t)\right)\right\|_{\infty} \lesssim \int_{0}^{t} \frac{1}{(t-\tau)^{1 / 2 \alpha_{1}}}\left\|b_{1}(\tau)\right\|_{\infty}\|z(\tau)\|_{\infty} \mathrm{d} \tau$

$$
\begin{aligned}
& \lesssim r^{1-\beta_{1}-\beta_{2}} \int_{0}^{t}(t-\tau)^{-\frac{1}{2 \alpha_{1}}} \mathrm{~d} \tau \sup _{0<\tau<t}\|z(\tau)\|_{\infty} \\
& \lesssim r^{1-\beta_{1}-\beta_{2}} t^{1-\frac{1}{2 \alpha_{1}}} \sup _{0<\tau<t}\|z(\tau)\|_{\infty},
\end{aligned}
$$

$\left\|\mathcal{B}_{\alpha_{1}}(y(t), y(t))\right\|_{\infty}+\left\|\mathcal{B}_{\alpha_{1}}(z(t), z(t))\right\|_{\infty}$

$$
\begin{aligned}
& \lesssim \int_{0}^{t} \frac{1}{(t-\tau)^{1 / 2 \alpha_{1}}}\left(\|y(\tau)\|_{\infty}^{2}+\|z(\tau)\|_{\infty}^{2}\right) \mathrm{d} \tau \\
& \lesssim t^{1-\frac{1}{2 \alpha_{1}}}\left(\sup _{0<\tau<t}\|y(\tau)\|_{\infty}^{2}+\sup _{0<\tau<t}\|z(\tau)\|_{\infty}^{2}\right) .
\end{aligned}
$$

Thus we have

$$
\begin{aligned}
\|y(t)\|_{\infty} \lesssim & r^{1-\beta_{1}-2 \beta_{2}} t^{1-\frac{1}{2 \alpha_{1}}-\frac{\theta_{2}}{2 \alpha_{1}}}+r^{2\left(1-\beta_{1}-\beta_{2}\right)} t^{1-\frac{1}{2 \alpha_{1}}} \\
& +r^{-\beta_{1}} t^{1-\frac{1}{2 \alpha_{1}}-\frac{\theta_{1}}{2 \alpha_{1}}} \sup _{0<\tau<t}\|y(\tau)\|_{\infty}+r^{-\beta_{2}} t^{1-\frac{1}{2 \alpha_{1}}-\frac{\theta_{2}}{2 \alpha_{1}}} \sup _{0<\tau<t}\|z(\tau)\|_{\infty} \\
& +r^{1-\beta_{1}-\beta_{2}} t^{1-\frac{1}{2 \alpha_{1}}} \sup _{0<\tau<t}\|z(\tau)\|_{\infty} \\
& +t^{1-\frac{1}{2 \alpha_{1}}}\left(\sup _{0<\tau<t}\|y(\tau)\|_{\infty}^{2}+\sup _{0<\tau<t}\|z(\tau)\|_{\infty}^{2}\right) .
\end{aligned}
$$

By (3.12), (4.27) and the fact $u_{1}=0$, we have

$$
\begin{aligned}
\|z(t)\|_{\infty} \lesssim & \left\|\mathcal{B}_{\alpha_{1}}\left(\mathrm{e}^{-t(-\Delta)^{\alpha_{2}}} u_{0}, b_{1}(t)\right)\right\|_{\infty}+\left\|\mathcal{B}_{\alpha_{2}}\left(\mathrm{e}^{-t(-\Delta)^{\alpha_{2}}} u_{0}, z(t)\right)\right\|_{\infty} \\
& +\left\|\mathcal{B}_{\alpha_{2}}\left(\mathrm{e}^{-t(-\Delta)^{\alpha_{2}}} b_{0}, y(t)\right)\right\|_{\infty}+\left\|\mathcal{B}_{\alpha_{2}}\left(b_{1}(t), y(t)\right)\right\|_{\infty}+\left\|\mathcal{B}_{\alpha_{2}}(y(t), z(t))\right\|_{\infty} .
\end{aligned}
$$

For $\alpha_{2}>1 / 2$ and $0<\frac{\theta_{1}}{2 \alpha_{2}}, \frac{\theta_{2}}{2 \alpha_{2}}<1$, a similar calculation shows that

$$
\begin{aligned}
\|z(t)\|_{\infty} \lesssim & r^{1-2 \beta_{1}-\beta_{2}} t^{1-\frac{1}{2 \alpha_{2}}-\frac{\theta_{1}}{2 \alpha_{2}}}+r^{-\beta_{1}} t^{1-\frac{1}{2 \alpha_{2}}-\frac{\theta_{1}}{2 \alpha_{2}}} \sup _{0<\tau<t}\|z(\tau)\|_{\infty} \\
& +r^{-\beta_{2}} t^{1-\frac{1}{2 \alpha_{2}}-\frac{\theta_{2}}{2 \alpha_{2}}} \sup _{0<\tau<t}\|y(\tau)\|_{\infty}+r^{1-\beta_{1}-\beta_{2}} t^{1-\frac{1}{2 \alpha_{2}}} \sup _{0<\tau<t}\|y(\tau)\|_{\infty} \\
& +t^{1-\frac{1}{2 \alpha_{2}}} \sup _{0<\tau<t}\|y(\tau)\|_{\infty} \sup _{0<\tau<t}\|z(\tau)\|_{\infty} .
\end{aligned}
$$

Let $w(t)=\|y(t)\|_{\infty}+\|z(t)\|_{\infty}$ and $w=\sup _{0<\tau<t}\|y(\tau)\|_{\infty}+\sup _{0<\tau<t}\|z(\tau)\|_{\infty}$. Adding (4.30) and (4.31) yields

$$
\begin{aligned}
w(t) \lesssim & r^{1-\beta_{1}-2 \beta_{2}} t^{1-\frac{1}{2 \alpha_{1}}-\frac{\theta_{2}}{2 \alpha_{1}}}+r^{2\left(1-\beta_{1}-\beta_{2}\right)} t^{1-\frac{1}{2 \alpha_{1}}}+r^{1-2 \beta_{1}-\beta_{2}} t^{1-\frac{1}{2 \alpha_{2}}-\frac{\theta_{1}}{2 \alpha_{2}}} \\
& +\left(r^{-\beta_{1}} t^{1-\frac{1}{2 \alpha_{1}}-\frac{\theta_{1}}{2 \alpha_{1}}}+r^{-\beta_{2}} t^{1-\frac{1}{2 \alpha_{2}}-\frac{\theta_{2}}{2 \alpha_{2}}}+r^{1-\beta_{1}-\beta_{2}} t^{1-\frac{1}{2 \alpha_{2}}}\right) \sup _{0<\tau<t}\|y(\tau)\|_{\infty} \\
& +\left(r^{-\beta_{2}} t^{1-\frac{1}{2 \alpha_{1}}-\frac{\theta_{2}}{2 \alpha_{1}}}+r^{1-\beta_{1}-\beta_{2}} t^{1-\frac{1}{2 \alpha_{1}}}+r^{-\beta_{1}} t^{1-\frac{1}{2 \alpha_{2}}-\frac{\theta_{1}}{2 \alpha_{2}}}\right) \sup _{0<\tau<t}\|z(\tau)\|_{\infty} \\
& +\left(t^{1-\frac{1}{2 \alpha_{1}}}+t^{1-\frac{1}{2 \alpha_{2}}}\right) w^{2}
\end{aligned}
$$




$$
\begin{aligned}
& :=C_{1}+C_{2} \sup _{0<\tau<t}\|y(\tau)\|_{\infty}+C_{3} \sup _{0<\tau<t}\|z(\tau)\|_{\infty}+C_{4} w^{2} \\
& \lesssim C_{1}+\left(C_{2}+C_{3}\right) w+C_{4} w^{2} \\
& =C_{1}+\left(C_{2}+C_{3}+C_{4} w\right) w .
\end{aligned}
$$

We shall choose large enough $r$ and small enough $T>0$, such that

$$
C_{0}:=C_{2}+C_{3}+C_{1} C_{4} \ll 1
$$

for $0 \leqslant t \leqslant T$. We claim the following estimate holds:

$$
\|w(t)\|_{\infty} \lesssim C_{1} \lesssim r^{1-\beta_{1}-2 \beta_{2}} t^{1-\frac{1}{2 \alpha_{1}}-\frac{\theta_{2}}{2 \alpha_{1}}}+r^{2\left(1-\beta_{1}-\beta_{2}\right)} t^{1-\frac{1}{2 \alpha_{1}}}+r^{1-2 \beta_{1}-\beta_{2}} t^{1-\frac{1}{2 \alpha_{2}}-\frac{\theta_{1}}{2 \alpha_{2}}},
$$

for all $0<t \leqslant T$. Indeed, since $w(0)=0$, and $w(t)$ is continuous, $\|w(t)\|_{\infty}$ cannot cross $2 C_{1}$. Otherwise, at certain time $t_{0}$, we have $w\left(t_{0}\right)=2 C_{1}$. Combining with (4.32), it implies

$$
2 C_{1} \lesssim C_{1}+2\left(C_{2}+C_{3}+2 C_{1} C_{4}\right) C_{1}
$$

and hence

$$
C_{2}+C_{3}+C_{1} C_{4} \gtrsim 1
$$

which contradicts $(4.33)$.

Now we choose $r$ and $T$ to guarantee (4.33). Note that the powers of $t$ in $C_{0}$ are all non-negative by the hypothesis of the lemma. Thus,

$C_{0} \lesssim r^{1-\beta_{1}-\beta_{2}} T^{1-\frac{1}{2 \alpha_{2}}}+r^{1-\beta_{1}-\beta_{2}} T^{1-\frac{1}{2 \alpha_{1}}}+r^{2\left(1-\beta_{1}-\beta_{2}\right)} T^{1-\frac{1}{2 \alpha_{1}}}\left(T^{1-\frac{1}{2 \alpha_{1}}}+T^{1-\frac{1}{2 \alpha_{2}}}\right)$.

Let $T=r^{-\gamma}$. It follows

$C_{0} \lesssim r^{1-\beta_{1}-\beta_{2}-\gamma\left(1-\frac{1}{2 \alpha_{2}}\right)}+r^{1-\beta_{1}-\beta_{2}-\gamma\left(1-\frac{1}{2 \alpha_{1}}\right)}+r^{2\left(1-\beta_{1}-\beta_{2}\right)-\gamma\left(2-\frac{1}{\alpha_{1}}\right)}+r^{2\left(1-\beta_{1}-\beta_{2}\right)-\gamma\left(2-\frac{1}{2 \alpha_{1}}-\frac{1}{2 \alpha_{2}}\right)}$.

We choose $\gamma$ such that

$$
\gamma>\frac{1-\beta_{1}-\beta_{2}}{1-1 /\left(2 \alpha_{1}\right)}, \quad \gamma>\frac{1-\beta_{1}-\beta_{2}}{1-1 /\left(2 \alpha_{2}\right)},
$$

which guarantees all the powers of $r$ are negative in (4.34). Hence (4.33) is satisfied for $r$ large enough. It proves the conclusion of the lemma.

\subsection{Finishing the proof}

Now we are ready to complete the proof of theorem 1.1. Since $u_{0}$ and $b_{0}$ are smooth and space-periodic, there exists $T^{*}>0$ and a smooth space-periodic solution $(u(t), b(t))$ to (1.1) on $\left[0, T^{*}\right)$ with $u(0)=u_{0}, b(0)=b_{0}$, such that either $T^{*}=+\infty$ or

$$
\limsup _{t \rightarrow T^{*}-}\left(\|u(t)\|_{\infty}+\|b(t)\|_{\infty}\right)=+\infty .
$$

Lemmas 4.4, 4.5, and 4.6 imply that $T^{*}>T$. Now using (3.6), we combine the imbedding estimate (2.2), lemmas $4.3,4.4,4.5$ and 4.6 to obtain that, for $\left|k_{1}\right|^{-2 \alpha_{2}} \leqslant t \leqslant T$

$$
\begin{aligned}
& \|b(\cdot, t)\|_{\dot{B}_{\infty, \infty}^{-s}} \geqslant\left\|b_{10}(\cdot, t)\right\|_{\dot{B}_{\infty, \infty}^{-s}}-\left\|b_{11}(\cdot, t)\right\|_{\infty}-\left\|b_{12}(\cdot, t)\right\|_{\infty}-\left\|\mathrm{e}^{-t(-\Delta)^{\alpha_{2}}} b_{0}\right\|_{\infty}-\|z(\cdot, t)\|_{\infty} \\
& \gtrsim r^{1-\beta_{1}-\beta_{2}}\left(1-r^{\beta_{1}-1} t^{-\frac{\theta_{2}}{2 \alpha_{2}}}-r^{-\beta_{2}} t^{1-\frac{1}{2 \alpha_{1}}-\frac{\theta_{2}}{2 \alpha_{1}}}-r^{1-\beta_{1}-\beta_{2}} t^{1-\frac{1}{2 \alpha_{1}}}-r^{-\beta_{1}} t^{1-\frac{1}{2 \alpha_{2}}-\frac{\theta_{1}}{2 \alpha_{2}}}\right) \\
& \gtrsim r^{1-\beta_{1}-\beta_{2}}\left(1-r^{\beta_{1}-1}\left|k_{1}\right|^{\theta_{2}}-r^{-\beta_{2}} T^{1-\frac{1}{2 \alpha_{1}}-\frac{\theta_{2}}{2 \alpha_{1}}}-r^{1-\beta_{1}-\beta_{2}} T^{1-\frac{1}{2 \alpha_{1}}}\right),
\end{aligned}
$$


where in the last step we used the fact $r^{-\beta_{1}} t^{1-\frac{1}{2 \alpha_{2}}-\frac{\theta_{1}}{2 \alpha_{2}}} \ll \frac{1}{2}$, since the power of $r$ is negative and the power of $t$ is non-negative. We will choose parameters so that

$$
A:=r^{\beta_{1}-1}\left|k_{1}\right|^{\theta_{2}}+r^{-\beta_{2}} T^{1-\frac{1}{2 \alpha_{1}}-\frac{\theta_{2}}{2 \alpha_{1}}}+r^{1-\beta_{1}-\beta_{2}} T^{1-\frac{1}{2 \alpha_{1}}} \leqslant 1 / 2 .
$$

Let $\left|k_{1}\right|=r^{\zeta}$ with positive $\zeta$, and $T=r^{-\gamma}$ as in lemma 4.6. Then

$$
A=r^{\beta_{1}-1+\zeta \theta_{2}}+r^{-\beta_{2}-\gamma\left(1-\frac{1}{2 \alpha_{1}}-\frac{\theta_{2}}{2 \alpha_{1}}\right)}+r^{1-\beta_{1}-\beta_{2}-\gamma\left(1-\frac{1}{2 \alpha_{1}}\right)} \text {. }
$$

To make (4.37) hold for large enough $r$, it is sufficient to choose $\zeta, \gamma$ such that

$$
\left\{\begin{array}{l}
0<\zeta<\frac{1-\beta_{1}}{\theta_{2}} \\
\frac{1-\beta_{1}-\beta_{2}}{1-1 /\left(2 \alpha_{1}\right)}<\gamma<2 \alpha_{2} \zeta \\
1-\frac{1}{2 \alpha_{1}}-\frac{\theta_{2}}{2 \alpha_{1}} \geqslant 0
\end{array}\right.
$$

Moreover, the condition $\gamma<2 \alpha_{2} \zeta$ in (4.38) guarantees that $\left|k_{1}\right|^{-2 \alpha_{2}}<T$, which is required in lemma 4.4. We verify that there exist $\beta_{1}, \beta_{2}, \theta_{1}$ and $\theta_{2}$ such that the assumption (4.28) in lemma 4.6 and conditions (4.35) (4.38) are compatible. Indeed, one can take $\beta_{1}=\beta_{2}=\frac{1}{2}-\epsilon$ for $0<\epsilon<\frac{1}{6}$. Since $\alpha_{1}, \alpha_{2} \geqslant 1$, it follows

$$
\frac{1-\beta_{1}-\beta_{2}}{1-1 /\left(2 \alpha_{1}\right)} \leqslant 4 \epsilon, \quad \frac{1-\beta_{1}-\beta_{2}}{1-1 /\left(2 \alpha_{2}\right)} \leqslant 4 \epsilon .
$$

On the other hand, due to the relation $\theta_{1}+\theta_{2}=2 \alpha_{2}$ in (4.28), we have

$$
2 \alpha_{2} \frac{1-\beta_{1}}{\theta_{2}}>1-\beta_{1}>\frac{1}{2}+\epsilon .
$$

Thus, the first two conditions in (4.38) are compatible. Straightforward computation shows all the other conditions among (4.28), (4.35) and (4.38) are also compatible.

Given any $\delta>0$ in theorem 1.1, we now choose a suitable large $r$ such that

$$
r^{1-\beta_{1}-\beta_{2}} \gtrsim \frac{1}{\delta}
$$

Therefore, it follows from (4.36) and (4.37) that

$$
\|u(\cdot, T)\|_{\dot{B}_{\infty, \infty}^{-s}} \gtrsim r^{1-\beta_{1}-\beta_{2}} \gtrsim \frac{1}{\delta} .
$$

Finally, lemma 4.2 implies that the initial data $u_{0}$ satisfies

$$
\left\|u_{0}\right\|_{\dot{B}_{\infty, \infty}^{-\theta_{1}}} \lesssim r^{-\beta_{1}} \lesssim \delta, \quad\left\|b_{0}\right\|_{\dot{B}_{\infty, \infty}^{-\theta_{2}}} \lesssim r^{-\beta_{2}} \lesssim \delta .
$$

This competes the proof of theorem 1.1.

\section{Acknowledgment}

The work of AC was partially supported by NSF Grant DMS-1108864.

\section{References}

[1] Bourgain J and Pavlović N 2008 Ill-posedness of the Navier-Stokes equations in a critical space in 3D J. Funct. Anal. 255 2233-47

[2] Cannone M 2004 Harmonic analysis tools for solving the incompressible Navier-Stokes equations Handbook of Mathematical Fluid Dynamics vol 3 (Amsterdam: North-Holland) pp 161-244 
[3] Cao C and Wu J 2011 Global regularity for the 2D MHD equations with mixed partial dissipation, magnetic diffusion Adv. Math. 226 1803-22

[4] Cheskidov A and Dai M 2014 Norm inflation for generalized Navier-Stokes equations Indiana J. Math. 63 869-84

[5] Cheskidov A and Shvydkoy R 2012 Ill-posedness for subcritical hyperdissipative Navier-Stokes equations in the largest critical spaces J. Math. Phys. 53115620

[6] Cheskidov A and Shvydkoy R 2010 Ill-posedness of basic equations of fluid dynamics in Besov spaces Proc. Am. Math. Soc. 138 1059-67

[7] Dai M, Qing J and Schonbek M E 2011 Norm inflation for incompressible Magneto-hydrodynamic system in $\dot{B}_{\infty}^{-1, \infty}$ Adv. Diff. Eqns $16725-46$

[8] Koch H and Tataru D 2001 Well posedness for the Navier-Stokes equations Adv. Math. 157 22-35

[9] Lemarié-Rieusset P G 2002 Recent Developments in the Navier-Stokes Problem (Chapman and Hall/CRC Research Notes in Mathematics vol 431) (Boca Raton, FL: Chapman and Hall/CRC)

[10] Schmeisser H-J and Triebel H 1987 Topics in Fourier Analysis and Function Spaces (Chichester: Wiley)

[11] Sermange M and Temam R 1983 Some mathematical questions related to the MHD equations Commun. Pure Appl. Math. 36 635-64

[12] Wu J 2003 Generalized MHD equations J. Diff. Eqns 195 284-312

[13] Wu J 2011 Global regularity for a class of generalized magnetohydrodynamic equations J. Math. Fluid Mech. 13 295-305

[14] Wu J 2008 Regularity criteria for the generalized MHD equations Commun. Partial Diff. Eqns 33 285-306

[15] Yoneda T 2010 Ill-posedness of the 3D-Navier-Stokes equations in a generalized Besov space near $B M O^{-1}$ J. Funct. Anal. 258 3376-87

[16] Yu X and Zhai Z 2012 Well-posedness for the fractional Navier-Stokes equations in the largest critical spaces $\dot{B}_{\infty, \infty}^{-(2 \beta-1)}$ Math. Methods Appl. Sci. 35 676-83 